\title{
Impact of Cloud Computing on ERP implementations in Higher Education
}

\author{
Ms. Shivani Goel, Dr Ravi Kiran, Dr Deepak Garg \\ Thapar University, Patiala
}

\begin{abstract}
Penetration of Higher Education in all regions is increasing all over the globe at a very fast pace. With the increase in the number of institutions offering higher education, ERP implementations has become one of the key ingredient to achieve competitiveness in the market. Many researchers have given their inputs specifying the different nature of ERP implementation in Educational institutions then the corporate organizations. Recently Cloud computing has become a buzzword and it is having applications in many domains. Researchers have already started applying cloud computing in ERP implementations of Higher education. This paper gives an insight into the nature of cloud computing impact on ERP implementations and discusses various issues related to this. Paper comes up with guidelines regarding the use of cloud computing technology in the ERP implementations of Higher Technical Education institutions.
\end{abstract}

Keywords- Cloud Computing; ERP; Higher Technical Education; ERP implementation.

\section{INTRODUCTION}

Cloud Computing has got different meaning to people working in different areas of computer science. Basically it is all about how the services are managed and delivered. Definitely it works on the principle of economies of scale at application, software and hardware level. It results

in many benefits like service provisioning, reduced costs, optimum resource utilization. Customer is now not tense about the robustness and continuity of software services in case of increasing demand of services embedded in cloud computing applications. Cloud handles elastic demand and the scalability limits go to infinity.

Good news is that good ERP systems should also have the characteristics fault tolerance, data sharing, privacy in communications and data handling, sharing data in diverse application modules.

Going a little deeper into both it will definitely strike in our mind that cloud computing technology can meet several of the advance and futuristic requirements of ERP implementations in organizations of higher technical education and can cater to increasing volume and range of services.[1]

Virtualization: It is related to autonomic computing in the context of utilizing the hardware or software resources including the processing power of a machine. There are different types of virtualizations like Hardware virtualization, Desktop virtualization, OS-level virtualization, Network virtualization and application virtualization etc. The similarity in all these is that virtualization is all about saving costs and running parallel virtual tasks from a single source increasing the utility of that source.
Service Oriented Architecture: In this kind of setting customer is the king. Customer is not aware of the heterogeneity of the different hardware architectures in the back of service providers. Customer is also not concerned with the different software applications running in different tiers of the web environment. SOA uses loose coupling concept and interfaces are defined in terms of protocols.[2]

SaaS (Software as a service) : In our context SaaS means that when the infrastructure need not to be developed in the organization but there is a service provider managing all the infrastructure at some remote location and providing the services in real time through a thin client to the users of the organization.

Ubiquitous computing: With this traditional desktop model has been left behind and users can now access the services through the device of their choice. It can be a mobile phone, ipad or any other thin client. The users are many times not even aware of this that they are using ubiquitous computing. Young generation now has grown and has left behind the desktop era.[3]

\section{EVOLUTION OF ERP IMPLEMENTATIONS IN HIGHER EDUCATION INSTITUTIONS}

\section{A. Traditional ERP implementation}

Still more than half of the institutions in the developing countries are following traditional approach due to different reasons. In these kinds of implementations different modules like admissions, fee collection, attendance, grading, Feedback system, Billing, Smart classes, Inventory, Human resource management etc are implemented in an Adhoc manner without having an overall objective of a comprehensive ERP. Many of the modules are generally developed at different times and these modules don't talk to each other. There is no concept of service architecture being used in these kinds of implementations. Cloud computing theory is out of scope for these kinds of implementations.

\section{B. On-Campus implementation}

Most of the implementations in the 90 s stick to this category, where organisations have developed in-house infrastructure for ERP implementations and don't have any outside dependence.

\section{Implementations with Internet Host Provider}

In these kinds of implementations virtualization, Server side scripts or tools, Storage and networking component is looked after by the host who has been hired for providing the 
services. It leads to lower cost of hardware by the organization. It also dilutes the requirement of highly skilled professions to

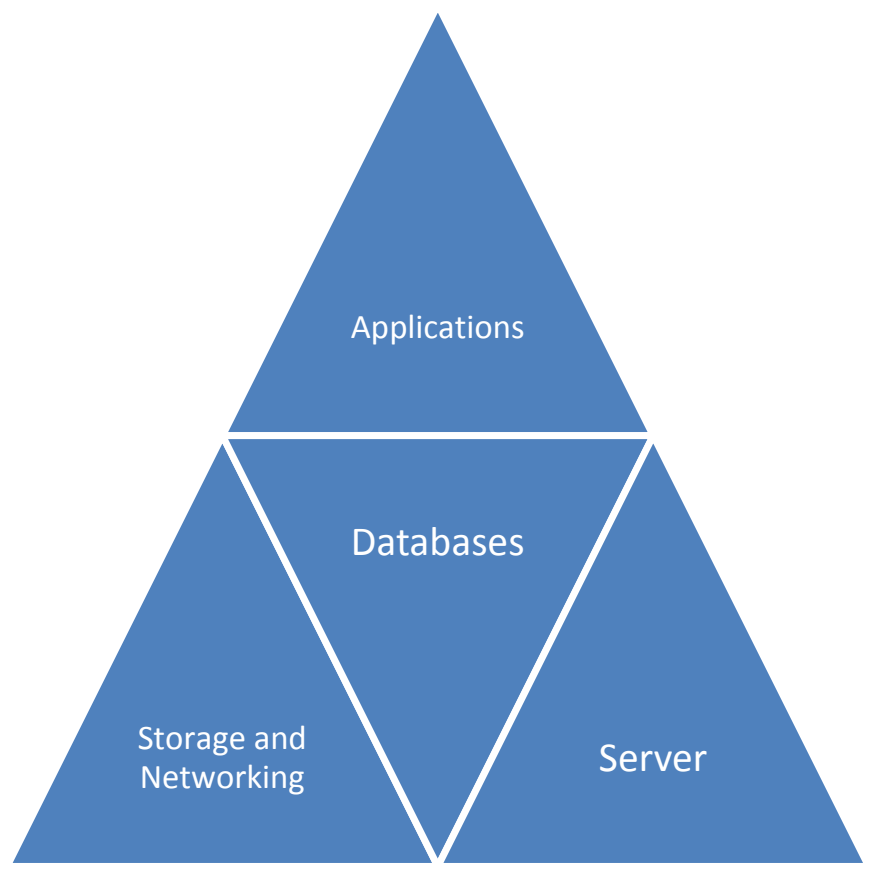

Fig 1.1 Cloud Components

maintain the system. Databases and applications are still run and managed at the campus. These implementations take some advantages of ubiquitous computing and SoA but bottlenecks still remain.[4]

\section{Cloud implementations}

Apart from the applications part everything is managed by the cloud server provider. Applications are anyway to be installed or made available at the user end. Applications can run in different variety of devices like TV, mobile phones, web kiosks, ipads, laptops etc. Databases, Servers, networking and storage components are still handled by the cloud provider.

\section{BENEFITS OF CLOUD COMPUTING IN ERP IMPLEMENTATIONS AT HIGHER TECHNICAL EDUCATION INSTITUTIONS[5-8]}

- Reduced cost: Whole of the hardware is now the responsibility of the cloud service provider. There is no requirement of any hardware at the campus, whatsoever. Even the cost of software decreases. You don't need to buy the fix time licenses or user based licenses. Now you only need to pay based on the usage of a particular service.

- Unfettered Access: All the faculty members, students, parents, management, recruiters, vendors can access the system according to the privileges granted to them. The access can be through any wired or wireless protocols using multiple devices of their choice.
- Security: Standard encryption and decryption techniques will be used and there is no need to worry about the security of the applications.

- Uptime: Almost zero downtime can be expected. However it may also depend upon the trust factor of your service provider and the feedback from the existing customers.

- Manpower: No manpower is required to be recruited at the institution.

- Futuristic Needs: Scalability, extension, peak load performance work becomes very simple. Generally this kind of support is available with every cloud provider.

- Customization: Any institution is not bound to use all the services being provided in the cloud application. The customer can choose the services based on specific requirements and the budgetary allocations.

- Group organizations: It is even more cost effective for institutions which have multiple courses and multiple campuses at different places. The access is all in real time through the web through any explorer like safari, Mozilla or IE.

- Integration : It can also integrate with the biometric, fingerprint, swipe and other machines already working in your organisations.

- There is no additional risk in case of any emergency or natural disasters because of third party involvement.The abbreviation "i.e." means "that is", and the abbreviation "e.g." means "for example".

\section{Challenges in IMPlementing Cloud Computing FOR ERP IMPLEMENTATION IN HIGHER TECHNICAL EDUCATION[9-12]}

- Elasticity Complexity: The demands and services in the cloud computing environment are elastic and integrated with the inherent distributed and parallel architectural characteristics. It involves highly skilled technical labour to develop and maintain such applications.

- Superstructure Emergence: Cloud is emerging as a superstructure and bypassing the boundaries of functional and vertical domains. Cloud server hosting different domain applications may become an issue because of overlapping demand and supply requirements.

- Technological Bottlenecks : Emergence of clouds also require corresponding technology upgradation in terms of new data structures for handling massive and dynamic data, new file systems, storage technologies etc.

- Serializability and consistency: Clouds are servicing the users in 24-hr format from different regions. We need to address the issue of trade-off between the consistency and serializability in sophisticated applications. 
- Programming model: New Programming paradigms are need of the hour which offers something between virtualization and physical reality.

- Monitoring, Analysis and Building Trust: Due to the scale of the applications running and the criticality of the services constant monitoring of the cloud servers for different parameters of operation is required. Regular reporting after proper analysis is required, which will in turn help in building the trust of the users in cloud systems.

- Mobility and provisioning: Most of the users are now accessing the services through the devices which are fast moving. So it requires the integration of cutting edge mobile routing technologies with the cloud services technologies.

\section{CONCLUSION}

It has been established that cloud computing will be helpful in improving the cost, maintenance and technical efficiency of ERP implementations in technical education institutions. However, it is seen that apart from the benefits there are many issues which need to be dealt with. The issues can be overcome with proper planning. The association of right people at the time the system is evolving is very crucial.

\section{REFERENCES}

[1] Brian Hayes. 2008. Cloud computing. Commun. ACM 51, 7 (July 2008), 9-11

[2] Mache Creeger. 2009. CTO Roundtable: Cloud Computing. Queue 7, 5, Pages 1 (June 2009), 2 pages.

[3] Aaron Weiss. 2007. Computing in the clouds. netWorker 11, 4 (December 2007), 16-25
[4] Kemal A. Delic and Martin Anthony Walker. 2008. Emergence of the Academic Computing Clouds. Ubiquity 2008, August, Article 1 (August 2008)

[5] Sanjay Ghemawat, Howard Gobioff, and Shun-Tak Leung. 2003. The Google file system. In Proceedings of the nineteenth ACM symposium on Operating systems principles (SOSP '03). ACM, New York, NY, USA, 29-43.

[6] Roxana Geambasu, Cherie Cheung, Alexander Moshchuk, Steven D. Gribble, and Henry M. Levy. 2008. Organizing and sharing distributed personal web-service data. In Proceeding of the 17th international conference on World Wide Web (WWW '08). ACM, New York, NY, USA, 755-764.

[7] Michael Vrable, Stefan Savage, and Geoffrey M. Voelker. 2009. Cumulus: Filesystem backup to the cloud. Trans. Storage 5, 4, Article 14 (December 2009), 28 pages.

[8] Michael Isard and Yuan Yu. 2009. Distributed data-parallel computing using a high-level programming language. In Proceedings of the 35th SIGMOD international conference on Management of data (SIGMOD '09), Carsten Binnig and Benoit Dageville (Eds.). ACM, New York, NY, USA, 987-994.

[9] Faouzi Kamoun. 2009. Virtualizing the Datacenter Without Compromising Server Performance. Ubiquity 2009, August, pages

[10] Jeanna Matthews, Tal Garfinkel, Christofer Hoff, and Jeff Wheeler. 2009. Virtual machine contracts for datacenter and cloud computing environments. In Proceedings of the 1st workshop on Automated control for datacenters and clouds (ACDC '09). ACM, New York, NY, USA, 25-30

[11] Harold C. Lim, Shivnath Babu, Jeffrey S. Chase, and Sujay S. Parekh. 2009. Automated control in cloud computing: challenges and opportunities. In Proceedings of the 1st workshop on Automated control for datacenters and clouds (ACDC '09). ACM, New York, NY, USA, 13-18.

[12] Albert Greenberg, James Hamilton, David A. Maltz, and Parveen Patel. 2008. The cost of a cloud: research problems in data center networks. SIGCOMM Comput. Commun. Rev. 39, 1 (December 2008), 68-73. 